\title{
Análise multitemporal do uso do solo da microbacia do Rio Sarandi no Sudoeste do estado do Paraná, Brasil utilizando imagens LANDSAT/TM
}

\author{
Multitemporal analysis of land use in the watershed of the River Sarandi in Southwest of the \\ Paraná State, Brazil using LANDSAT/TM
}

\author{
Clóvis Cechim Júnior \\ Universidade Estadual do Oeste do Paraná, Cascavel, Paraná, Brasil
}

Mara Rubia Silva

Universidade Federal da Integração Latino-Americana, Foz do Iguaçu, Paraná, Brasil

\section{Resumo}

A avaliação das alterações que um determinado local sofre ao longo de vários anos pode ser efetuada a partir da análise de imagens em diferentes periodos. Este trabalho teve como objetivo realizar a análise multitemporal do uso do solo da microbacia do Rio Sarandi que possui predomínio de agricultura familiar e forte potencial agropecuário e está localizada na mesorregião Sudoeste do estado do Paraná, região Sul do Brasil. Para verificar as mudanças de uso do solo da bacia foi necessário aplicar a técnica de realce linear de imagem para melhorar visualmente a informação espectral e facilitar a interpretação das imagens. Fez-se o estudo com imagens do satélite LANDSAT/ TM no periodo de 1985 a 2010, com a técnica de classificação supervisionada com o classificador Bhattacharya. Os dados foram processados no programa SPRING e com os resultados obtidos foi possível evidenciar que ao longo desses 25 anos houve uma diminuição na cobertura florestal de 0,40\%, de 4,21\% nas áreas de pastagens e de 6,64\% para solo exposto e um aumento de 10,52\% na agricultura, de 0,62\% na área urbana e de 0,11\% em lâmina de água. A avaliação da acurácia dos mapeamentos gerados foi feita usando como referência as imagens LANDSA/TM, o IK variou entre (97,2 e 98,7\%) e o EG médio foi de 98\% o que indica a eficiência do classificador.

Palavras-chave: Imagens orbitais. Classificação digital. Interpretação de imagens.

\section{Abstract}

The evaluation of the changes a particular location undergoes over several years may be carried out with the analysis of images in different periods of time. The objective of this study was to carry out the multi-temporal analysis of the land use on the Sarandi River watershed that has predominance of family farms and high agricultural potential and is located in the Southwest mesoregion of the state of Paraná, Southern Brazil. To verify the changes of land use in the basin was necessary to apply the linear image enhancement technique to improve spectral information and facilitate images interpretation. The classification method used was supervised as the Bhattacharya classifier, on LANDSAT/ TM satellite's images, from 1985 to 2010. The data were processed by SPRING and the results showed along these 25 years a $0.40 \%$ decline in forest cover, with decrease in grazing areas in a ratio of $4.21 \%$ and around $6.64 \%$ for exposed soil and a increase of $10.52 \%$ in agriculture, $0.62 \%$ in urban area and $0.11 \%$ in water blade. The evaluation of the accuracy of the mappings generated was made using as reference the images LANDSA/TM, IK ranged between (97.2 98.7\%) and EG medium was 98\% which indicates the efficiency of the classifier. 


\section{1 introdução}

O desenvolvimento urbano e o crescimento populacional têm gerado grandes alterações ao ambiente. A busca de novos métodos que evidenciam a situação dos recursos naturais e que permitem analisar suas alterações com a possibilidade de promover novos cenários, é de extrema significância para o conhecimento do uso desses recursos, atendendo a demanda atual sem comprometer as futuras gerações.

A utilização de geotecnologias associados ao geoprocessamento, Sistemas de Informações Geográficas (SIG) e Sensoriamento Remoto destaca-se, pelo fato de considerar também a importância das ações voltadas à conservação dos ecossistemas e a preservação biodiversidade.

As ferramentas utilizadas no Geoprocessamento permitem identificar a origem dos aspectos modificadores do ambiente e a intensidade destas alterações, bem como os processos de degradação ambiental, tratando-se de bacias hidrográficas.

Os SIGs permitem armazenar e manipular informações geográficas e analisar dados de sensoriamento remoto, sendo imprescindíveis em monitoramentos ambientais e na caracterização de impactos ambientais em bacias hidrográficas que são unidades fisiográficas de planejamento.

"Uma das vantagens de se utilizar o Sensoriamento Remoto para interpretação do uso do solo e cobertura vegetal natural é que as informações podem ser atualizadas devido à característica de repetitividade de aquisição das imagens" (Brito \& Prudente, 2005).

As microbacias formam as bacias hidrográficas assim como as mesmas são as regiões mais suscetíveis aos aspectos que ocasionam os processos de impactos ambientais e a existência dos mesmos, o uso de microbacias como unidade de estudo permite identificar a degradação sobre o uso do solo e a vegetação.

O mapeamento é fundamental para os estudos de uso e ocupação dos solos para o manejo e planejamento da gestão ambiental destes, pois auxiliam na determinação dos impactos ambientais causados pela atividade antrópica e em suas modificações. Além disso, também auxiliam no monitoramento de fenômenos climáticos, desta forma, muitos são os trabalhos que fazem uso de analise temporal de imagens (Piroli et al., 2002; Disperati et al., 2003; Arai et al., 2011; Torres, 2011; Wondie et al., 2011; Marchesan et al., 2013; Scott et al., 2014; Tran et al., 2015).

Neste contexto, este trabalho teve como objetivo estimar os processos de degradação e modificações do uso do solo da microbacia do Rio Sarandi, situada no Sudoeste Paranaense. Utilizando a análise multitemporal de imagens do satélite Land Remote Sensing Satellite (LANDSAT) série 5, nos períodos compreendidos entre 1985 a 2010, avaliou-se a variação e a quantificação das áreas de uso de solo presentes na microbacia.

\section{2 Área, materiais e métodos}

A microbacia do Rio Sarandi pertence à bacia hidrográfica do Rio Iguaçu na unidade hidrográfica de gerenciamento de

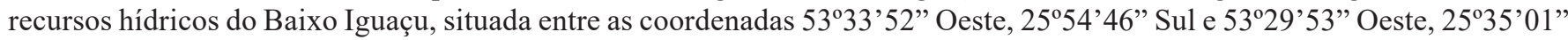
Sul, possui uma área de 22.762,06 ha (Fig. 1).

\subsection{Localização da área}

A microbacia do Rio Sarandi como área de estudo destaca-se pelo fato de abranger três municípios da região sudoeste do estado do Paraná, Realeza, Santa Izabel do Oeste e Ampére, com um manancial de abastecimento público a montante que é responsável pelo abastecimento dos municípios de Realeza e Santa Izabel do Oeste.

Figura 1. Mapa de localização da microbacia do Rio Sarandi.

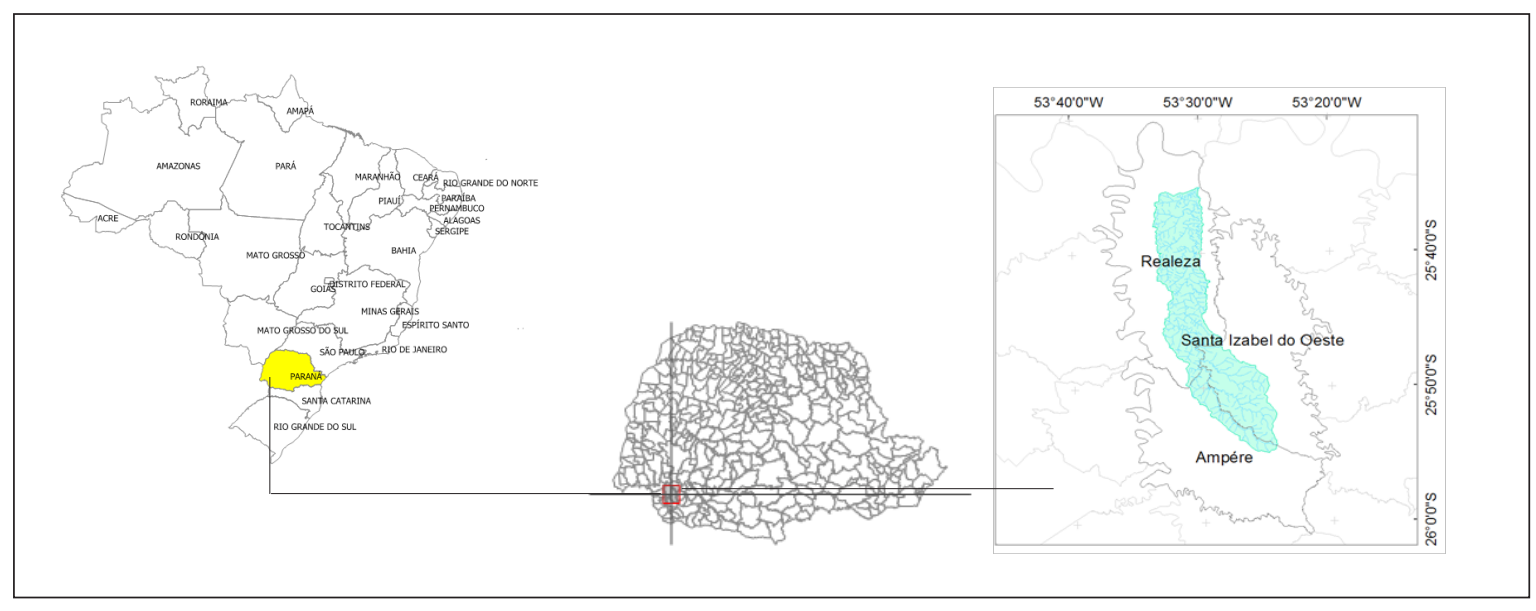


Além de haver predomínio de agricultura familiar à constituição de solo proporciona alta aptidão agrícola para produção de grãos, apesar das desvantagens em relação à mecanização devido ao relevo desfavorável, o sudoeste alcançou significativa inserção de representatividade na dinâmica do agronegócio.

A região destaca-se no contexto estadual como a segunda maior em produção de aves e suínos e com importante contribuição na produção de soja e milho, particularizando-se pela dependência de atividades agropecuárias (IPARDES, 2006).

A figura 2 evidencia os dados da população censitária e sua evolução nos anos de 2006 a 2010, nos municípios integrantes da microbacia do Rio Sarandi.

Figura 2 - Evolução populacional dos municípios integrantes da microbacia Sarandi (conforme AMSOP, 2011).

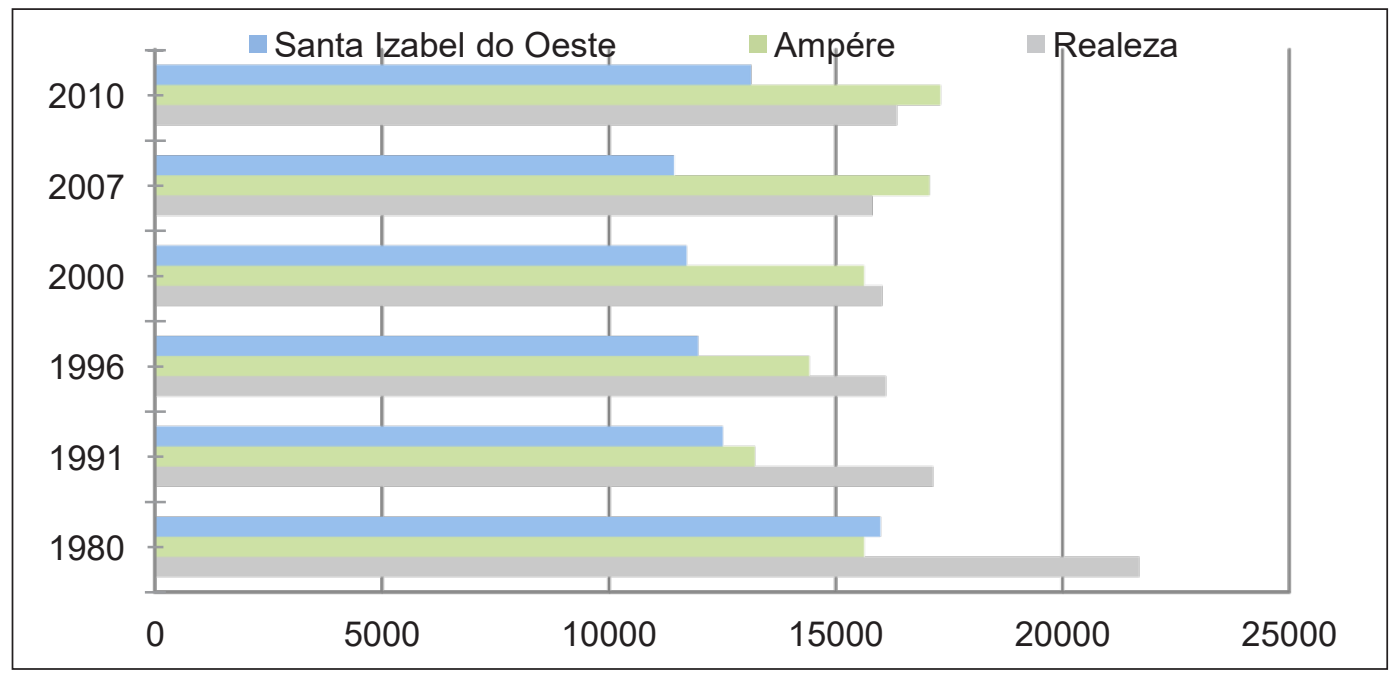

\subsection{Materiais}

A metodologia deste trabalho incluiu revisão bibliográfica, aquisição de base cartográfica e imagens satelitais, levantamento de dados referentes a solo, vegetação e clima da microbacia para a geração de documentos cartográficos.

As ferramentas computacionais utilizadas para a realização deste trabalho foram o Sistema Operacional Windows 7; Software Sistema de Processamento de Informações Georreferenciadas - SPRING, versão 5.1.8, e suas extensões IMPIMA 5.1.8 e SCARTA 5.1.8 de domínio público, desenvolvido pelo Instituto Nacional de Pesquisas Espaciais (INPE).

Como base cartográfica foram usados a Base hidrográfica em formato vetorial na escala 1:50.000, cedidas pelo Instituto das Águas do Estado do Paraná e pelo Instituto de Terras Cartografia e Geociências (ITCG); Base Hidrográfica Unificada, fornecido pela COPEL/Aguasparaná, 2011; contendo a Altimetria, Rede Hídrica e Ottobacias; Divisão Político-Administrativa do Paraná, (ITCG, 2010); Mapas de Solos e de clima - Estado do Paraná (ITCG, 2008).

Para a elaboração deste trabalho, foram utilizados como dados matriciais as imagens de diferentes datas do satélite LANDSAT-5 compreendidas para a área de estudo datadas entre 1985 e 2010 (Tabela 1).

Tabela 1- Imagens utilizadas na pesquisa

\begin{tabular}{c|c|c|c|c}
\hline Data & Satélite & Sensor & Órbita/Ponto & Resolução Espacial \\
\hline $02-10-1985$ & & & & \\
$09-05-1990$ & & & & \\
$04-10-1995$ & LANDSAT 5 & TM & $223 / 78$ & \\
$02-09-2000$ & & & & \\
$01-06-2005$ & & & & \\
\hline
\end{tabular}

As imagens foram obtidas no catálogo de imagens do (INPE, 2016) em épocas distintas, o uso das imagens em períodos distintos se justifica pelo fato de que nem todas as imagens disponíveis apresentam boa visibilidade em relação à cobertura de nuvens o que influenciaria no processamento digital das imagens.

Outro fator utilizado para facilitar a interpretação visual e processamento das imagens foi sua aquisição nos meses de período de entressafra como maio e inicio de junho, setembro e inicio de outubro, nestes períodos o zoneamento agrícola é evidenciado pelo término das colheitas e inicio das plantações para os novos cultivos. 


\subsection{Métodos}

Os procedimentos e as etapas realizados para a execução da metodologia de pesquisa estão descritos na (Fig. 3).

Figura 3 - Fluxograma dos procedimentos metodológicos

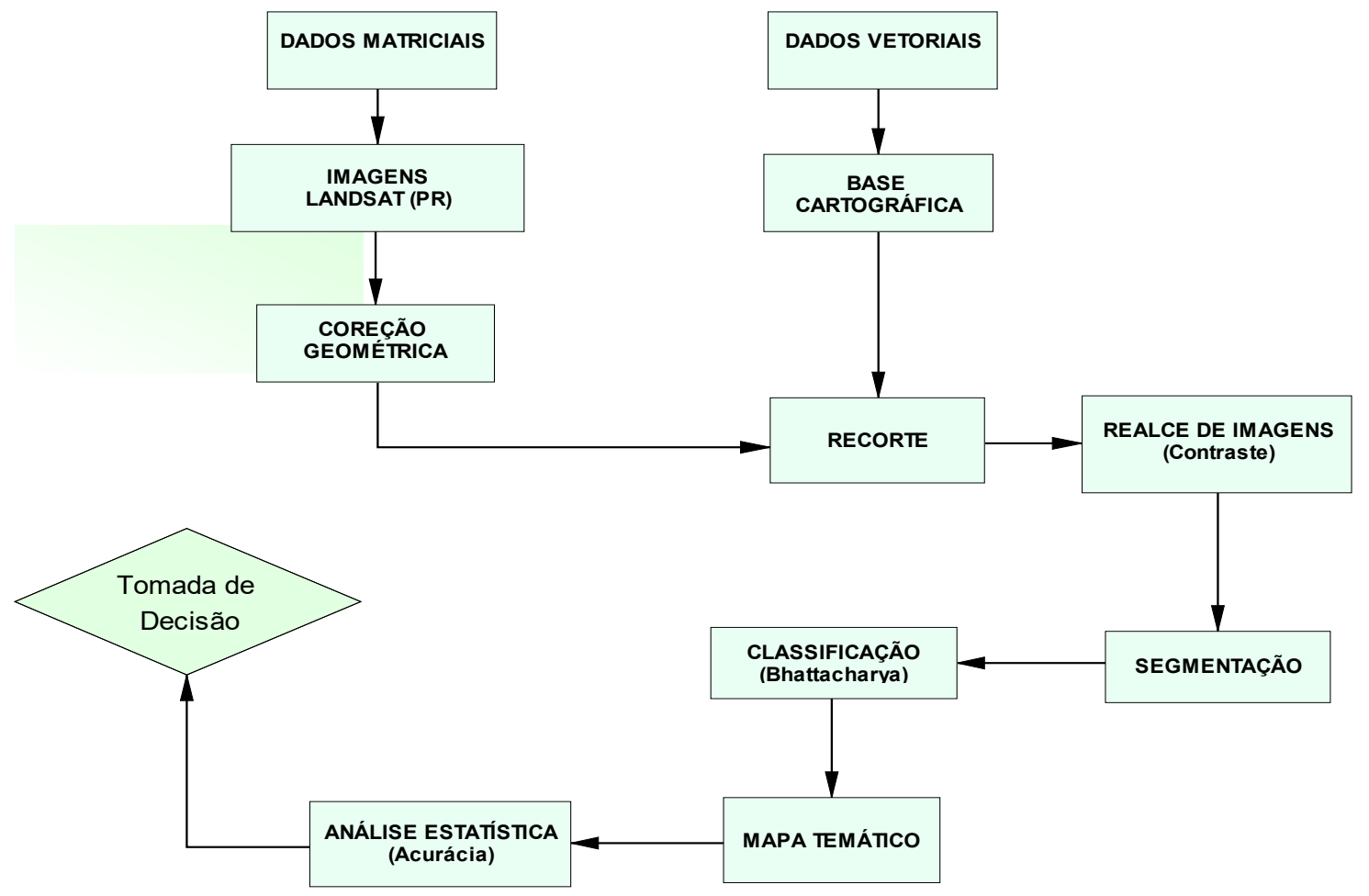

\subsubsection{Pré processamento de imagens}

A primeira etapa consistiu na aquisição de dados matriciais (raster) e vetoriais, organização e criação do banco de dados, com a criação do projeto, o qual define a área física do trabalho é o local em que os dados ficam armazenados em planos de informações (PI).

A segunda etapa consistiu no ajuste geométrico ou registro das imagens da bacia utilizando os dados vetoriais do ITCG. O ajuste foi realizado por pontos de controle no processo (mapa-imagem), em que o mapa foi à referência e a imagem LANDSAT a imagem a ser ajustada, a imagem de 1985 foi a primeira imagem a ser ajustada, processo mapa-imagem e para as outras foi utilizado o processo (imagem-imagem). Este procedimento foi repetido para todas as imagens.

Mesmo procedimento realizado por Arai et al. (2011) em sua técnica de multirresolução multitemporal para detecção e mapeamento do desflorestamento na Amazônia Brasileira, que precisou corrigir geometricamente as imagens LANDSAT-7/ETM+ usando pontos de controle selecionados manualmente, obtendo uma raiz resultante do erro quadrático inferior a 0,5 pixels.

A terceira etapa foi composta pelo recorte das cenas do satélite pelas bandas espectrais $3,4,5$ com a resolução espacial de $30 \mathrm{~m}$ da órbita 223 ponto 78 . As seis imagens (1985 a 2010) foram recortadas usando como máscara o uso do limite da Bacia Hidrográfica do Rio Sarandi, para o processo foi usado à função recortar plano de informação ferramenta presente no SIG SPRING (Santos et al., 2010).

\subsubsection{Processamento digital de imagens}

A quarta etapa consistiu na aplicação do realce das imagens LANDSAT usando a técnica de contraste. A operação consiste na seleção da banda a ser realçada aplicando a distribuição da respectiva cor com auxilio do histograma visualizado durante a operação.

Foi usado a composição de falsa cor onde foi selecionado a banda 3 no canal azul, a banda 4 no verde e a banda 5 no vermelho, composição R(5)G(4)B(3). A mesma composição usada por Piroli et al. (2002) em análise do uso da terra 
na microbacia Arroio do Meio na região sul. Torres (2011) e Marchesan et al. (2013) também utilizaram a mesma composição de bandas espectrais para avaliar o uso da terra na microbacia do Arroio Grande e no município de Faxinal do Soturno. Segundo Disperati et al. (2003) a composição colorida falsa cor é muito utilizada para realizar estudos que envolvem avaliações de recursos ambientais.

A quinta parte do trabalho consistiu na segmentação das imagens por crescimento de regiões, o procedimento consiste em classificar cada "pixel" com propriedades semelhantes em uma mesma região espectralmente homogênea, sendo incluídos nestas propriedades os níveis de cinza, textura e cor que são elementos presentes nas imagens.

Foram testadas varias áreas de pixel e similaridade dentre estas 4 e 6,6 e 10,10 e 14,12 e 14,12 e 16,14 e 16,20 e 22, este processo foi realizado no menu imagem função segmentação onde foi selecionado as bandas da imagem recortada e aplicado a área de pixel e de similaridade.

\subsubsection{Classificação digital de imagens}

A sexta fase consistiu no processo de classificação de imagens pelo classificador de Bhattacharya que faz uso de treinamento supervisionado com elemento de classificação de região. Definiu-se os nomes dos treinamentos ou as classes de classificação, como: agricultura, água, floresta, pastagem, solo exposto e urbanização.

Com a classe selecionado adquiriu-se a amostragem na região da imagem criada pela segmentação, cada região constitui um número de pixels. Adquirida as amostragens das classes na função de treinamento foi elaborada a classificação da imagem sendo definido o limiar de aceitação.

O limiar de aceitação indica a porcentagem (\%) de "pixels" da distribuição de probabilidade de uma classe do treinamento ser classificada como pertencente à mesma (DPI, 2006). O limiar usado foi de 99,9\% o que significa que 99,9 \% da imagem classificada englobaram 99,9\% de "pixels" enquanto houve uma rejeição de $0,01 \%$.

A partir das classes de uso de solo existentes foram determinadas suas variações por meio da conversão do mapeamento de classes para imagem temática, sendo definida a categoria e a junção entre temas e classes. A finalidade da conversão é determinar a análise das medidas das classes, onde foram calculadas as áreas das respectivas classes de uso do solo.

\subsubsection{Análises de desempenho do classificador}

A última etapa consistiu na determinação da acurácia da classificação de Bhattacharya quanto à referência (verdade terrestre), sendo usado o conjunto amostras adquirido nas imagens para a avalição, para o procedimento foi usada à matriz de confusão para determinar o índice Kappa (IK) e Exatidão Global (EG) (Congalton, 1991; Congalton \& Green, 1999), a matriz permite representar se o classificador confundiu as amostras de pixel semelhantes adquiridos com alguma das classes de uso do solo.

Como resultado gerou-se os mapeamentos feitos usando o modelo de classificação Bhattacharya para o período compreendido entre os anos de 1985 a 2010 na microbacia do Rio Sarandi (Fig. 4).

A análise de evolução espacial do uso do solo da microbacia Rio Sarandi demonstra que houve aumento de áreas em ha de cultivos agrícolas, comparando com Wondie et al. (2011) em seu trabalho sobre as mudanças espaciais e temporais da dinâmica de cobertura do solo no Parque Nacional das Montanhas Simien localizado no Nordeste da Etiópia.

Na ocasião ao longo do período de 1984 e 2003, evidenciou como tendências principais de evolução a diminuição de áreas agrícolas e aumento de áreas florestais passando de 11,71\% em 1984 para 15,63\% em 2003. A precisão e o valor do IK das classificações variaram de $88 \%$ a $85 \%$ respectivamente.

Corroborando com os resultados encontrados por Tran et al. (2015) na avaliação dinâmica de mudanças de cobertura de uso do solo no Delta do Mekong nos últimos 40 anos com o litoral do distrito da província de Ca Mau, Vietnã entre 1973 a 2011 com uso de imagens (LANDSAT e SPOT), demonstrou que houve redução em áreas de terras cultivadas durante o período, no entanto a classe é a mais predominante.

Durante o período de 38 anos, as áreas de terras cultivadas, terras sem cultivos e florestas de manguezais, teve a maior redução absoluta na área com 155, 111 e $67 \mathrm{Km}^{2}$, respectivamente. As exatidões globais nos diferentes períodos de tempo variaram de $81 \%$ a $89 \%$ e os índices gerais de IK de 0,77 para 0,87 . A acurácia aumentou para a maioria dos períodos recentes, devido à maior resolução espacial de imagens de satélite e disponibilidade de conjuntos de dados de referência mais detalhados e precisos. Parte superior do formulário 
Figura 4 - Mapeamentos de uso do solo da microbacia do Rio Sarandi. A) Ano 1985; B) Ano 1990; C) Ano 1995; D) Ano 2000; E) Ano 2005; F) Ano 2010

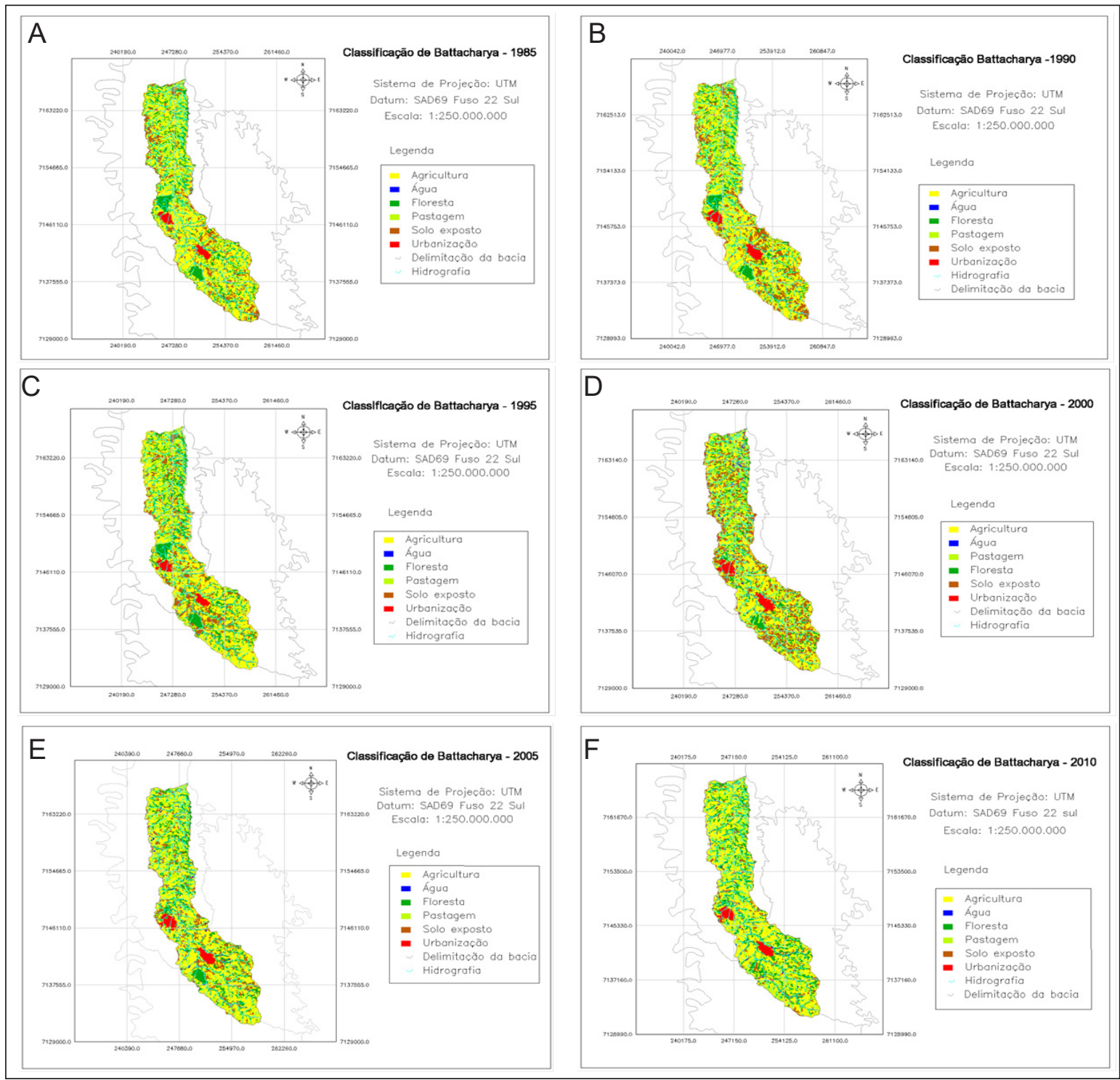

A quantificação do uso do solo em ha por ano obtida a partir da análise multitemporal de dados LANDSAT é evidenciada na Tabela 2.

Tabela 2 - Quantificação do uso do solo em ha por ano

\begin{tabular}{lccccccc}
\hline Classes & 1985 & 1990 & 1995 & 2000 & 2005 & 2010 & Variação \\
\hline Agricultura & $11.286,05$ & $9.478,96$ & $9.522,50$ & $10.878,29$ & $12.926,74$ & $13.680,11$ & $10,52 \%$ \\
Água & 0,34 & 18,96 & 11,42 & 19,90 & 20,20 & 25,64 & $0,11 \%$ \\
Floresta & $4.491,01$ & $3.586,21$ & $4.039,91$ & $3.332,74$ & $4.150,02$ & $4.399,05$ & $-0,40 \%$ \\
Pastagem & $3.977,38$ & $5.353,84$ & $4.347,16$ & $4.270,08$ & $3.297,82$ & $3.018,02$ & $-4,21 \%$ \\
Solo exposto & $2.518,74$ & $3.840,30$ & $4.356,26$ & $3.699,79$ & $1.757,78$ & $1.008,30$ & $-6,64 \%$ \\
Urbanização & 488,54 & 483,78 & 484,81 & 561,27 & 609,49 & 630,94 & $0,62 \%$ \\
Total & $22.762,06$ & $22.762,06$ & $22.762,06$ & $22.762,06$ & $22.762,06$ & $22.762,06$ & - \\
\hline
\end{tabular}




\section{Discussão dos resultados}

Couto et al. (2011) avaliaram as mudanças que ocorreram na cobertura vegetal da planície do rio Paraná, no período entre 1976 e 2007 a partir de dados de Sensoriamento Remoto constataram que áreas ocupadas por corpos de água e solo exposto apresentaram aumento enquanto as variações de vegetação de áreas úmidas e vegetação arbustiva e gramínea apresentaram pequena diminuição, inferindo que a ação antrópica sobre a planície foi ampliada.

Resultados estes semelhantes aos encontrados na evolução da classe de água presentes na microbacia do Rio Sarandi, com pequena inferência antrópica devido ao aumento de áreas urbanizadas (Fig. 5).

Com os resultados evidenciados no gráfico é possível observar que a classe agricultura sempre se mostrou presente em maior quantidade em ha/ano durante todos os períodos analisados, entre 1985 a 2010 houve aumento de 10,52\% totalizando 2.394,06 ha.

Figura 5 - Gráfico de evolução multitemporal do uso do solo

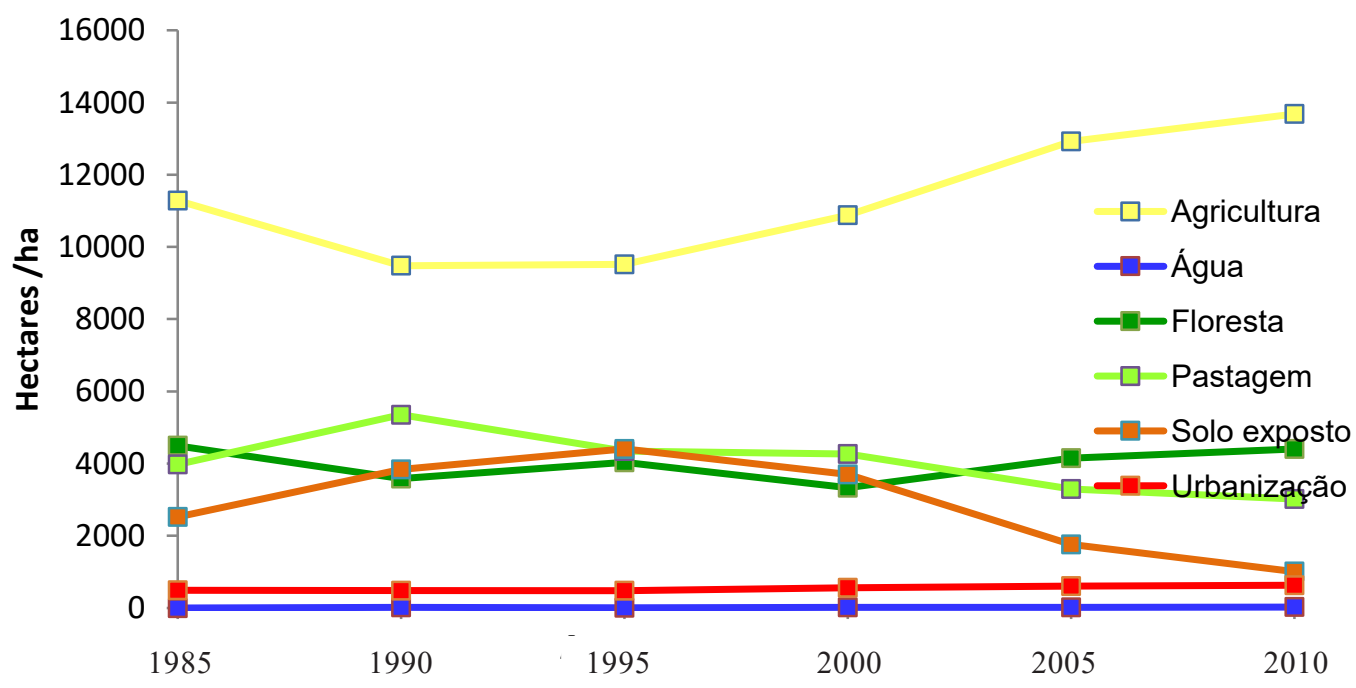

As classes que tiveram maior estabilidade foram às classes de água e urbanização que representaram um pequeno aumento na ordem de 25,30 ha $0,11 \%$ para água e 142,40 ha para a urbanização o que corresponde a $0,62 \%$.

A classe de floresta teve situações de variações durante todo o decorrer da análise temporal, totalizando uma diminuição na faixa de $0,40 \%$ o que corresponde a 91,60 ha.

Diminuição também encontrada na análise de temporalidade da cobertura do solo na bacia do Rio Negro, MS de Bachega et al. (2009) que apresentava em 1985, 20\% de áreas de mata e em 2007 constatou 15\%, concluindo houve um desmatamento na bacia, sendo este maior do que o observado na microbacia Sarandi.

As classes de pastagem e solo exposto tiveram variação com o passar dos anos, com o final da análise no ano de 2010 essas classes diminuíram 959,36 ha 4,21\% e 1.510,44 ha o equivalente a 6,64\% respectivamente que passaram a ser usados para cultivos agrícolas.

Os resultados encontrados para a classe pastagens e para classe de lâmina de água se assemelham com os resultados obtidos por Torres (2011) e com o aumento de área urbana por Scott et al. (2014) para quantificar a composição física da morfologia urbana do País de Gales baseado na série temporal (1989 - 2011) com imagens LANDSAT TM/ETM+.

Quanto à classe de lâmina de água, apesar da variação durante as épocas do ano observável em imagens de messes mais frio que apresenta maior nível pluviométrico e seco com pouca chuva, para a microbacia do Rio Sarandi houve um aumento na proporção de $0,11 \%$ o que equivale a 25,64 ha para o mês de junho de 2010 .

O trabalho realizado por Scott et al. (2014) evidenciou Áreas de Superfície Impermeável (ISA) tendo as maiores mudanças ocorrendo durante (1989-1999), onde a área urbana aumentou consistentemente em quantidades variáveis.

Embora as extensões exteriores de áreas urbanas do País de Gales não alteraram ao longo de 22 anos, o ISA contido dentro deles expandiu significativamente, o que deixa supor que políticas de restrições urbana levaram ao preenchimento das fronteiras existentes urbanas e os espaços urbanos dentro deles, a fim de acomodar a expansão da população.

Este trabalho permite a compreensão dos padrões de organização do espaço agrícola, e suas alterações pela ação antrópica, bem como para o estabelecimento de medidas de preservação, conservação e recuperação da microbacia do Rio Sarandi e para o estabelecimento de um planejamento ambiental.

Com a quantificação das classes de uso do solo percebem-se problemáticas ambientais como a degradação do solo, ausência 
de mata ciliar em trechos do afluente e uso excessivo de agrotóxicos na agricultura, que ocupa cerca de $60 \%$ da microbacia correspondendo a mais da metade da área total, resultado obtido para o ano de 2010 a partir do processamento digital das imagens.

Com a geração dos mapas temáticos foi possível obter agilidade na visualização e entendimento para a determinação das condições em que se encontrava a microbacia e os fatores causadores de impacto ambiental.

As imagens classificadas apresentaram uma variação de erro na seleção das amostras que compuseram as classes de 95 a 98\% na representação da realidade. Resultado semelhante encontrado por Peluzio et al. (2011) em seu estudo sobre a avaliação de diferentes algoritmos de classificação supervisionada, para o mapeamento da cobertura e uso da terra na bacia hidrográfica do Rio Alegre, ES mediante a utilização do aplicativo computacional SPRING.

Na ocasião obteve como resultado um índice de exatidão variando de 90 a $97 \%$ para o algoritmo de classificação supervisionado de Bhattacharya, assemelhando-se não somente na determinação das classes presentes na microbacia do Rio Sarandi mais também pelo fato de ser caracterizada pela prática de agricultura familiar, pecuária de subsistência e com cobertura do solo bastante heterogênea.

A acurácia obtida a partir da determinação do IK foi de 0,95 no ano de 1985, 0,98 no ano de 1990, 0,96 nos anos de 2000 e 2005 e 0,95 em Bhattacharya 2010, o que indica uma excelente qualidade temática gerada pela classificação e pelo EG com valores de $97,5 \%$ no ano de $1985,98,7 \%$ no ano de $1990,98,3 \%$ nos anos de 2000 e 2005 e $97,2 \%$ no ano de 2010 . Com relação à exatidão global, segundo Foody (2002), é desejável que uma classificação alcance índices de acerto superiores a $85 \%$, conforme situação confirmada pelo EG médio obtido nas classificações por Bhattacharya na microbacia do Rio Sarandi, com 98\%.

Como sugestão para futuros trabalhos nesta área recomenda-se o uso de técnicas de fusão de imagens com a finalidade de testar se há diferença entre as classificações e se as mesmas permitem uma melhor visualização das problemáticas ambientais e também a caracterização de subclasses da classe agricultura para representar a agricultura familiar na microbacia.

\section{Conclusões}

Com as técnicas de processamento de imagens, classificação digital e segmentação realizadas no SIG SPRING foi possível determinar e quantificar o uso do solo da microbacia do Rio Sarandi no sudoeste paranaense, de forma rápida e eficiente. E também foi possível verificar às alterações nas coberturas de uso do solo bem como nas áreas florestais em relação à influência de atividades antrópicas.

Dentre as alterações observadas, a área de remanescentes florestais de mata atlântica diminui no período avaliado de 1985 a 2010. A supressão de mata nativa em maior parte se deu para cultivos agrícolas conforme constatado na avaliação de acurácia analisada para toda a série temporal.

Verificou-se que a área de remanescentes florestais representou 19,34\% do total da microbacia em 2010, o que é inferior à taxa de área de reserva legal determinada na legislação ambiental para propriedades rurais.

A ausência de informações e pesquisas em microbacia hidrográficas dificulta ações dos governos municipais e estaduais no planejamento e na organização adequada do uso do solo, impossibilitando o monitoramento dos impactos e da degradação dos recursos naturais, tais como água e solo.

Sugere-se então, a adoção de medidas de intervenção de ações públicas com a inclusão de técnicas de manejo adequadas, como recuperação da cobertura florestal, APPs e áreas fragilizadas mais suscetíveis a impactos ambientais, podendo também ser introduzindo a prática da agroecologia à agricultura familiar inserida na microbacia.

\section{Agradecimentos}

Os autores são gratos ao Departamento de Sistemas de informações e Geoprocessamento do Instituto Águas Paraná pelo fornecimento da base cartográfica da bacia hidrográfica do Rio Sarandi.

\section{Referências}

AMSOP. Associação dos Municípios do Sudoeste do Paraná. 2011. Banco de dados do sudoeste do Paraná. Sudoeste - População censitária 1980 - 2010. 2011. Disponível em: <http://www.amsop.com.br/sudoeste.php?lc=censitaria.html.>. Acesso em: 10 nov. 2012.

ARAI E, Shimabukuro YE, Pereira G, Vijaykumar NLA. Multi-temporal technique for detecting and mapping deforestation in the Brazilian Amazon rainforest. Remote Sensing. 2011;3(12):1943-1956.

Bachega CCF, Paranhos Filho AC, Tiviroli VA, Miranda CS, Anache JAA. Análise multitemporal da cobertura do solo na bacia do Rio Negro, MS. In: XIV Simpósio Brasileiro de Sensoriamento Remoto; 2009 abr 25-30; Natal, Brasil. p. $1253-1259$. 
Brito JLS, Prudente DT. Análise temporal do uso do solo e cobertura vegetal do município de Uberlândia - MG, utilizando imagens ETM+ / LANDSAT 7. Sociedade e Natureza. 2005;17(32):37-46.

Couto EV, Souza filho EE, Hayakawa EH. Análise das modificações da cobertura vegetal da planície fluvial do alto rio Paraná no período entre 1976 e 2007. Acta Scientiarum Technology. 2011;33(2):205-213.

Disperati AA, Oliveira Filho PC, Bobrowski R, Dlugosz FL. Análise temporal da cobertura florestal do município de Irati - PR, utilizando imagens satelitárias LANDSAT. Revista Ciências Exatas e Naturais. 2003;5(1): 115-123.

Congalton RG. A review of assessing the accuracy of classifications of remotely sensed data. Remote Sensing of Environment. 1991;37(1):35-46.

Congalton RG, Green K. Assessing the accuracy of remotely sensed data: principles and practices. Boca Raton: Crc Press; 1999. 160p.

Foody GM. Status of land cover classification accuracy assesment. Remote Sensing of Environment. 2002;80(1): 185-201.

INPE. Instituto Nacional de Pesquisas Espaciais. 2016. Catálogo de Imagens. Disponível em: < http://www.dgi.inpe.br/CDSR/>. Acesso em: 18 abr. 2016.

ITCG. Instituto de Terras e Cartografia. 2008. Mapas e informações cartográficas do Estado do Paraná. Produtos cartográficos. Disponível em: <http://www.itcg.pr.gov.br/modules/conteudo/conteudo.php?conteudo=47.html>. Acesso em: 06 mai. 2012.

ITCG. Instituto de Terras e Cartografia. 2010. Mapas e informações cartográficas do Estado do Paraná. Produtos cartográficos. Disponível em: <http://www.itcg.pr.gov.br/modules/conteudo/conteudo.php?conteudo=47.html>. Acesso em: 07 mai. 2012.

IPARDES. Instituto Paranaense de Desenvolvimento Econômico e Social. Leituras Regionais: Mesorregião Geográfica Sudoeste Paraense/Instituto de Desenvolvimento Econômico e Social. Curitiba: BRDE; 2004.140 p.

IPARDES. Instituto Paranaense de Desenvolvimento Econômico e Social. Referências ambientais e socioeconômicas para uso do território do estado do Paraná: Uma contribuição ao zoneamento ecológico-econômico - ZEE. Curitiba: IPARDES; 2006. 160p.

IPARDES. Instituto Paranaense de Desenvolvimento Econômico e Social. Caderno estatístico município de Ampére Curitiba: IPARDES; 2012. 29p.

IPARDES. Instituto Paranaense de Desenvolvimento Econômico e Social. Caderno estatístico município de Realeza. Curitiba: IPARDES; 2011. 28p.

IPARDES. Instituto Paranaense de Desenvolvimento Econômico e Social. Caderno estatístico município de Santa Izabel do Oeste. Curitiba: IPARDES; 2012. 29p.

Marchesan J, Goergen LC, Nunes MM, Brites DI, Silva CK, Pereira RS. Análise multitemporal do uso e cobertura da terra no município de Faxinal do Soturno-RS nos anos de 1986, 1996, 2006 e 2011. Revista do Centro de Ciências Naturais e Exatas - UFSM, Santa Maria, Revista Eletrônica em Gestão, Educação e Tecnologia Ambiental. 2013;13(13):2785-2794.

Peluzio TMO, Saito NS, Klippel VH, Souza SM, Santos AR. Utilização de algoritmos de classificação supervisionada no mapeamento do uso e cobertura da terra no aplicativo computacional Spring 5.1.6. In: XV Simpósio Brasileiro de Sensoriamento Remoto - SBSR; 2011 mai 30-5; Curitiba, Brasil. p7808-7814.

Piroli EL, Becker ELS, Bolfe L, Pereira RS. Análise do uso da terra na microbacia do Arroio do Meio - Santa Maria - RS, por Sistemas de Informações Geográficas e imagens de satélite. Ciência Rural. 2002; 3(32):1678-4596.

Santos AR, Peluzio TMO, Saito NS. SPRING 5.1.2 passo a passo aplicações práticas. Alegre: UFES; 2010.153 p.

Scott D, Petropoulos GP, Moxley J, Malcolm H. Quantifying the physical composition of urban morphology throughout Wales based on the time series (1989-2011) analysis of Landsat TM/ETM+ images and supporting GIS Data. Remote Sensing. 2014;6(12):11731-11752. 
Wondie M, Schneider W, Melesse AM, Teketay D. Spatial and temporal land cover changes in the Simen Mountains National Park, a world Heritage site in Northwestern Ethiopia. Remote Sensing. 2011;3(12): 752-766.

Tran H, Tran T, Kervyn M. Dynamics of land cover/land use changes in the Mekong Delta, 1973-2011: A remote sensing analysis of the Tran Van Thoi District, Ca Mau Province, Vietnam. Remote Sensing. 2015;7(3): 2899-2925.

Torres DR. Análise multitemporal do uso da terra e cobertura florestal com dados dos satélites Landsat e Alos [dissertation]. Santa Maria: Programa de Pós-graduação em Engenharia Florestal, Centro de Ciências Rurais/ UFSM; 2011.97 p.

Torres SELMG, Souza RF, Saraiva AGS, Guimarães CL, Gadelha AG. Análise multitemporal da cobertura vegetal da bacia hidrográfica Cantinho - Itaporanga - PB. In: III Simpósio Brasileiro de Ciências Geodésicas e Tecnologias da Geoinformação; 2010 jul 27-30; Recife, Brasil. p. 00-008.

\section{Clóvis Cechim Júnior}

Universidade Estadual do Oeste do Paraná, Cascavel, Paraná, Brasil e-mail:juniorcechim@hotmail.com Participação do autor:

\section{Mara Rubia Silva}

Universidade Federal da Integração Latino-Americana, Foz do Iguaçu, Paraná, Brasil

e-mail: mara.silva@unila.edu.br

Participação do autor: 